\title{
Afrontamiento al estrés académico en estudiantes de Ciencias Médicas.
}

Coping with academic stress in students of Medical Sciences.

Liana Borges Sierra. ${ }^{1}$, Jorge César Cairo Martínez. ${ }^{2}$, María Magdalena Pedroso González. ${ }^{3}$ \& Luis Efraín Velastegui López. ${ }^{4}$

DOI: https://doi.org/10.33262/anatomiadigital.v4i2.1718

\begin{abstract}
.
Resumen.

Introduction: the term stress, one of the Introducción: el término stress, uno de most currently used by different health professionals, was introduced in 1926 by los más empleados actualmente por los diferentes profesionales de la salud, fue the Canadian doctor Hans Selye, who defined it as the body's general response to any stressor stimulus. Stress is one of the problems that most affects the physical and mental health of students, particularly in the Medicine career, where the study is characterized by a constant and growing academic demand that demands adaptation efforts from the student. Objective: to assess the stress introducido en 1926 por el médico canadiense Hans Selye, quien lo definió como la respuesta general del organismo ante cualquier estímulo estresor. El estrés es uno de los problemas que más afecta la salud física y mental de los estudiantes, en particular de la carrera de Medicina, donde el estudio se caracteriza por una constante y creciente exigencia académica que demandan del estudiante

\footnotetext{
${ }^{1}$ Facultad de Ciencias Médicas de Mayabeque, Mayabeque, Cuba, Estudiante de Tercer Año de Medicina, lianab_03@nauta.cu

${ }^{2}$ Facultad de Ciencias Médicas de Mayabeque, Mayabeque, Cuba, Estudiante de Quinto Año de Medicina, jorgecesar@infomed.sld.cu

${ }^{3}$ Facultad de Ciencias Médicas de Mayabeque, Mayabeque, Cuba, MSc. Medicina Bioenergética y Natural, Profesora Asistente, mpedroso@infomed.sld.cu

${ }^{4}$ Ciencia Digital Editorial, Ecuador, luisefrainvelastegui@ cienciadigital.org
} 
coping strategies that can be used by students of Medical Sciences. Method: a bibliographic review was carried out, using the resources available in the Infomed network, among which we can mention: SciELO and Ebesco, through the databases: Medline, Academic Search Premier and Medic Latina. Searches were conducted in English and Spanish. The Google Scholar search engine was used. The quality, reliability and methodological validity of the selected articles were analyzed to carry out an adequate review and current trends in the subject were assessed. Results: task overload, the type of work required, evaluations and exams are considered the main stressors in students, probably due to the decrease in time to carry out other activities of a family and social nature. There are various ways of coping with stress in Medical Sciences students, among them the following stand out: setting goals and priorities, managing time well, having an optimistic attitude and being systematic in studying. In addition to the Jacobson's progressive muscle relaxation method.

Keywords: stress, coping strategies, stressor esfuerzos de adaptación. Objetivo: valorar las estrategias de afrontamiento el estrés que pueden ser empleadas por los estudiantes de Ciencias Médicas.Método: se realizó una revisión bibliográfica, empleando los recursos disponibles en la red Infomed, entre los que podemos citar: SciELO y Ebesco, a través de las bases de datos: Medline, Academic Search Premier y Medic Latina. Las búsquedas se realizaron en inglés y español. Se utilizó el buscador Google Académico. Se analizó la calidad, fiabilidad y validez metodológica de los artículos seleccionados para realizar una adecuada revisión y se valoraron las tendencias actuales en la temática. Resultados: la sobrecarga de tareas, el tipo de trabajo requerido, las evaluaciones y los exámenes son considerados los principales factores estresantes en los educandos, probablemente se debe a la disminución del tiempo para realizar otras actividades de carácter familiar y social. Existen diversas maneras de afrontar el estrés en estudiantes de Ciencias Médicas, entre ellas destacan: establecer objetivos y prioridades, administrar bien el tiempo, tener una actitud optimista y ser sistemático en el estudio. Además del método de Relajación muscular progresiva de Jacobson.

Palabras claves: estrés, estrategias de afrontamiento, estresor.

\section{Introducción.}

Para muchos jóvenes y para sus familiares, estudiar una carrera universitaria representa una meta al considerar que obtener un grado académico incrementa las oportunidades laborales y, por ello, se está en posibilidades de aspirar a mejorar la calidad de vida. Para lograrlo, el ámbito universitario no sólo requiere que los jóvenes tengan interés en los 
estudios, sino que también deben mostrar buena organización, autonomía, habilidades específicas y capacidad para afrontar las situaciones escolares estresantes. (Barraza, A. (2014)

El término stress, uno de los más empleados actualmente por los diferentes profesionales de la salud, fue introducido en 1926 por el médico canadiense Hans Selye, quien lo definió como la respuesta general del organismo ante cualquier estímulo estresor. La palabra estrés, al igual que éxito, fracaso o felicidad, significan diferentes cosas para diferente gente, de tal manera que su definición es muy difícil. (Barraza, A. (2015)

El estrés es uno de los problemas de salud más generalizado en la sociedad actual. Es un fenómeno multivariable que resulta de la relación entre la persona y los eventos de su medio, los mismos que son evaluados como desmesurados o amenazantes para sus recursos y que ponen en riesgo su bienestar. Los tipos de estrés suelen ser clasificados tomando como base la fuente del estrés, en ese sentido se puede hablar del estrés amoroso y marital, del estrés sexual, del estrés familiar, del estrés por duelo, del estrés médico, del estrés ocupacional, del estrés militar, del estrés por tortura y encarcelamiento, y del estrés académico. (Bedoya, S.A., Perea, M. \& Ormeño, R. (2016)

Al decir de Orlandini, "Desde los grados preescolares hasta la educación universitaria de posgrado, cuando una persona está en un período de aprendizaje experimenta tensión. A ésta se le denomina estrés académico, y ocurre tanto en el estudio individual como en el aula escolar. El estrés académico es aquel que padecen los alumnos de educación media superior y superior y que tiene como fuente exclusiva a estresores relacionados con las actividades que van a desarrollar en el ámbito escolar.

Según Barraza Macias, "El estrés académico es un proceso sistémico de carácter adaptativo y esencialmente psicológico, que se presenta de manera descriptiva en tres momentos: primero, el alumno se ve sometido, en contextos escolares, a una serie de demandas que, bajo la valoración del propio alumno son consideradas estresores. Segundo, esos estresores provocan un desequilibrio sistémico (situación estresante), que se manifiesta en una serie de síntomas (indicadores del desequilibrio) y tercero, ese desequilibrio sistémico obliga al alumno a realizar acciones de afrontamiento para restaurar el equilibrio sistémico. El afrontamiento, término no tan popular como el de estrés, forma parte de los recursos psicológicos de cualquier individuo y es una de las variables personales declaradas como participantes en los niveles de calidad de vida, a la cual se atribuye un gran valor e importancia en las investigaciones sobre la calidad de vida y el bienestar psicológico. El afrontamiento "es el proceso a través del cual el individuo maneja las demandas de la relación individuo-ambiente que evalúa como estresantes y las emociones que ello genera" Los procesos de afrontamiento han sido estudiados por varios autores como son Lazarus, Font, Hernández y otros. Es por tanto uno de los conceptos más en boga en la investigación psicológica actual. En Cuba, el tema ha sido estudiado por el Dr. Orlandini, Dr. Clavijo Portieles, Zaldívar, quien plantea, "El afrontamiento al estrés, al ser positivo pudiera ser un factor protector de la salud y al ser negativo pudiera ser un factor de riesgo para enfermar ". 
Según Lazarus, "Los modos de afrontamiento al estrés serían las diferentes respuestas conductuales, cognitivas y emocionales empleadas para manejar y tolerar al mismo, permitiendo reducir la tensión y restablecer el equilibrio." No obstante, Lazarus y Folkman, plantean que afrontar no es equivalente a tener éxito, y piensan que tanto el afrontamiento como las defensas deben verse como algo que puede funcionar bien o mal en determinadas personas, contextos u ocasiones. (Caldera, J.F., Pulido, B.E. \& Martínez, M.G. (2017)

Los estudios de Medicina se caracterizan por una constante y creciente exigencia académica que demandan del estudiante esfuerzos de adaptación. A ello se agrega la necesidad de una preparación óptima, fundamental para el futuro profesional. Adicionalmente, la carrera se desarrolla en un periodo del ciclo vital con características que le son propias, la adolescencia y la adultez joven. Además, se postula que el estrés que soporta un estudiante de medicina durante su adiestramiento es obligadamente alto, con riesgo de menoscabo de su salud mental, ejemplo de ello lo constituye el estar sometidos a altos requisitos de memorización, a muchas horas al día estudiando, al estrés producido por las evaluaciones, a horarios irregulares de alimentación, descanso y convivencia familiar, lo que puede afectar su bienestar físico y psicológico, observándose en ellos alta prevalencia de estrés, correlacionado con algunos malestares tales como fatiga crónica, mala digestión, dolor abdominal, enfermedades alérgicas, hipertensión, ansiedad, depresión, mala concentración. El tema planteado se describe en diversas Facultades de Medicina del mundo. Algunos autores demandan mayor atención de ésta en el cuidado de la salud mental de sus estudiantes, muchos de los cuales encuentran la carrera muy estresante desde el inicio. En las Universidades norteamericanas de Tuffs y Harvard, se ha descrito que dos tercios del total de estresores que soportan los estudiantes de Medicina derivan específicamente de la vida académica y de los factores sociales a ella asociados. En la Universidad de Zimbabwe, en 109 estudiantes de primer año de Medicina, de 18 a 24 años de edad, Vaz y colectivo encontraron que el 64,5 porciento presentaba diversos niveles de estrés y depresión. En la Universidad de Toronto, Coburn y Jovaisas, en estudiantes del primer año de Medicina, encontraron que los factores académicos, especialmente aquellos vinculados a la auto percepción de posibles fracasos, constituían los estresores más significativos. (Celis, J., Bustamante, M., Cabrera, D., Cabrera, M., Alarcón, W. \& Monge, E. (2001)

\section{Metodologia.}

Se realizó una revisión bibliográfica, empleando los recursos disponibles en la red Infomed, entre los que podemos citar: SciELO y Ebesco, a través de las bases de datos: Medline, Academic Search Premier y Medic Latina. Las búsquedas se realizaron en inglés y español. Se utilizó el buscador Google Académico y se aplicó una estrategia de búsqueda utilizando las palabras claves y conectores: estrés académico, modos de afrontamiento, estrés en salud, estresores. Se analizó la calidad, fiabilidad y validez metodológica de los artículos seleccionados para realizar una adecuada revisión y se valoraron las tendencias actuales en la temática. 


\section{Resultados.}

El estrés académico es la reacción normal que tenemos frente a las diversas exigencias y demandas a las que nos enfrentamos en la Universidad, tales como pruebas, exámenes, trabajos, presentaciones, etc. Esta reacción nos activa y nos moviliza para responder con eficacia y conseguir nuestras metas y objetivos. Sin embargo, en ocasiones, podemos tener demasiadas exigencias al mismo tiempo, lo que puede agudizar la respuesta y disminuir nuestro rendimiento.

Según la posición de varios autores, como educadores, no se puede dejar de considerar que el ingreso, la permanencia y el egreso de un alumno de una institución educativa suele ser una experiencia de aprendizaje que va acompañada regularmente de estrés académico; esta afirmación, que para algunos puede ser casual, es respaldada por las investigaciones realizadas al respecto, y debe ser valorada como tal. (Feldman, L., Goncalves, L., ChacónPuignau, G., Zaragoza, J., Bagés, N. \& De Pablo, J. (2008)

Fisher y Hood (1986), demostraron que los estudiantes experimentan un incremento significativo en los niveles de depresión, síntomas obsesivos y pérdida de concentración tras seis semanas de permanencia en la universidad. Este medio académico demanda desempeños sobresalientes para alcanzar el éxito, y dicha exigencia implica para el ser humano un proceso de adaptación que puede resultar en la generación de estrés.

Igualmente, Mosley (1994), reporta en un estudio realizado en estudiantes de Medicina que, a mayores niveles de estrés en los estudiantes, éstos experimentan mayores niveles de depresión.

En tal contexto Pulido y Serrano (2011) quienes estudiaron distintas carreras universitarias, revelaron que los programas académicos de Psicología y Odontología pertenecientes al área de Ciencias de la Salud, logran obtener mayores índices de estrés académico a comparación con carreras de otros ámbitos como el caso de Contaduría y Derecho.

Díaz Clarisse, Ferreira Marta, Pérez Nohelia (2015). En cuanto a las situaciones generadoras de estrés se ha observado mayor porcentaje en los exámenes escritos y presentaciones orales, actividades que no pueden ser remplazadas o anuladas ya que las mismas se encontraron dentro de la actividad académica curricular.

Estresores académicos (González, J.L. (2001); Gutiérrez, Y.F., Londoño, K.F. \& Mazo, R. (2009)

Las exigencias que pueden dar origen al estrés son de dos tipos: Exigencias Internas, es decir, las que nos realizamos a nosotros mismos cuando somos muy autoexigentes, tenemos altas expectativas de logro, necesitamos mantener el control y nos esforzamos en ellos, entre otros; y las Exigencias Externas, es decir, las que nos va presentando nuestro entorno, dentro de las cuales destacan tener que entregar trabajos y rendir pruebas y exámenes en un tiempo acotado; realizar trabajos en grupo; exponer frente al curso 
(disertaciones); no entender contenidos abordados en la clase; tiempo limitado para las obligaciones académicas, entre otros.

Existen también una serie de situaciones vitales que nos estresan y que repercuten en nuestro estado emocional y físico, como: problemas legales, separación, matrimonio, embarazo, vacaciones, muerte de un amigo, cambio de residencia, cambio de hábito de dormir, reconciliación de pareja, lesión o enfermedad personal, cambio de hábitos alimentarios, despido del trabajo y/o cesantía, cambio de salud de un miembro de la familia, adicción y/o alcoholismo (propio o de cercano), incorporación de un nuevo miembro a la familia, comienzo o fin de etapa académica, cambio en las condiciones de vida, cambio de actividades sociales.

Inicialmente el estrés es normal y provechoso, ya que nos ayuda a activarnos para poder responder a las demandas y exigencias del medio, con el tiempo nuestro cuerpo y nuestra mente se van agotando y comienzan a disminuir en su rendimiento. (Lazarus, R.S. \& Folkman, S. (1986)

Fases del estrés. (Martín, I. M. (2015); Martínez, E.S. \& Díaz, D.A. (2017)

\section{Manifestaciones físicas}

Corresponden a los síntomas que se generan en nuestro cuerpo frente a los estresores habituales, algunos de ellos son: somnolencia (mayor necesidad de dormir), cansancio y fatiga, dolores de cabeza o jaquecas, dolor de estómago y/o color irritable, bruxismo (tensión de mandíbulas), dolor de espalda, taquicardia o palpitaciones fuertes, aumento o disminución del apetito, resfríos frecuentes (baja en el sistema inmune), dificultades para dormir (insomnio o pesadillas).

\section{Manifestaciones psicológicas y emocionales}

Son las reacciones emocionales y cognitivas que tenemos frente a situaciones de estrés, entre las que destacan: inquietud e hiperactividad, tristeza y/o desgano, ansiedad constante, dificultades para concentrarse, dificultades para pensar (quedarse en blanco), problemas de memoria, olvidos frecuentes, irritabilidad frecuente, temor a no poder cumplir con las obligaciones, baja motivación para actividades académicas, angustia y/o ganas de llorar frecuente.

\section{Manifestaciones conductuales}

Consisten en los cambios que experimentamos en nuestra conducta producto de la vivencia de situaciones de estrés, que son fácilmente observados por otros, entre los que podemos encontrar: frecuente tendencia a discutir con los demás, necesidad de estar solo, aislarse, uso de fármacos para estar despierto (auto medicados), preferir otras actividades por sobre el estudio, uso de fármacos para dormir (auto medicados), dificultad para sentarse a estudiar ("sacar la vuelta"), aumento del consumo de café y/o tabaco, dejar de asistir a clases, frecuente participación en carretes poco saludables, tendencia a dejar de hacer, por desgano o apatía, lo que se acostumbra normalmente. 


\section{Tipos de técnicas utilizadas para el estrés académico.}

Estrategias de afrontamiento: Es la percepción general asociada a cada una de las situaciones planteadas y que deriva en el afrontamiento del estrés. Entre las que tenemos: habilidad asertiva, elaboración y ejecución de un plan, elogios a sí mismo, la religiosidad, búsqueda de información sobre la situación y ventilación de la situación y confidencias. (Misra, R. \& Castillo, L. (2004); Pardo, J. (2017)

Algunas técnicas utilizadas para manejar el estrés académico son: (Pellicer, O., Salvador, A. \& Benet, I. (2015).

1-Administración del tiempo: La mala administración del tiempo puede provocar fatiga o apatía así como la sensación de no ser productivo, esto sucede cuando los estudiantes perciben un excesivo material de estudio en tan poco tiempo, para eso los estudiantes deberán establecer horarios de trabajo más realistas con horarios de descanso que puedan ser utilizados como espacio comodín ante situaciones imprevistas, además de priorizar los materiales de estudio según su relevancia en cuanto al tiempo de entrega y su importancia.

2-Establecer objetivos y prioridades: A pesar de poner en práctica la administración del tiempo para evitar sentirse abrumado con respecto a la carga de tareas la cual será muy útil, se debe ser realista y consciente de las posibilidades, así como las limitaciones. Es importante establecer prioridades y reconocer que algunas cosas no pueden hacerse en ese momento, priorizar es una habilidad que se debe aplicar en conjunto con la administración del tiempo.

Hacer una lista de todas las tareas, actividades y compromisos que tiene que hacer en un día. Seguidamente escribir la importancia de completar esta tarea, los beneficios, así como las consecuencias de no completarla.

3-Actitud positiva: Una actitud positiva puede ser la clave para llevar a cabo las anteriores técnicas y afrontar el estrés de manera general. Se sabe que nuestra predisposición mental, nuestras intenciones nos llevan a la dirección a la que queremos dirigirnos. Es decir, si tenemos una actitud positiva que saldremos de las situaciones estresantes, el humor nos brinda una situación de control y nos libera de las emociones negativas y desagradables.

El psicólogo Williams James planteó lo siguiente "no reímos porque somos felices, sino porque somos felices reímos". El humor es una herramienta que posibilita crear espacios de vinculación y generar un clima de confianza entre el grupo. Reír también contribuye a reducir la presión arterial, relaja los músculos, alivia el dolor, reduce las hormonas del estrés y dispara el sistema inmunológico. (Rodríguez, M. (s.f.)

Relajación muscular progresiva de Jacobson (Román, C. A., Ortiz, F. \& Hernández, Y. (2015); Sandín, B. (1995).

Esta técnica fue desarrollada por el psiquiatra estadounidense Edmund Jacobson en la década del '30. Su mecanismo de acción permite obtener el control voluntario de la 
tensión y la distensión muscular, permitiendo identificar la relación entre el estado muscular y el estado mental de tensión. Se le denomina "progresiva" ya que los avances se van consiguiendo de forma gradual. La forma de trabajo es ir tensando y relajando los diversos grupos musculares.

Piernas: extender los pies, elevar las piernas, presionarlas hacia abajo; estirar hasta punta del pie, tensionar los dedos y presionar los glúteos hacia abajo

Tronco: hundir y tensionar la zona abdominal; arquear la espalda manteniendo una respiración profunda; estirar y tensionar bíceps y tríceps y presionar los puños de las manos

Hombros: levantar hacia las orejas y llevarlos atrás

Cuello: llevar la cabeza hacia atrás; presionarla contra el pecho; inclinar la cabeza hacia los lados

Ojos: cerrarlos presionando; levantar las cejas tensionando frente y fruncir el ceño

Músculos del habla: levantar la comisura (sonrisa); arrugar los labios (beso); abrir al máximo y luego presionar las mandíbulas; estirar la lengua fuera de la boca, presionar contra el paladar y presionar en la zona inferior; y la nariz arrugarla hacia arriba.

\section{Conclusiones.}

- La sobrecarga de tareas, el tipo de trabajo requerido, las evaluaciones y los exámenes son considerados los principales factores estresantes en los educandos, probablemente se debe a la disminución del tiempo para realizar otras actividades de carácter familiar y social.

- Existen diversas maneras de afrontar el estrés en estudiantes de Ciencias Médicas, entre ellas destacan: establecer objetivos y prioridades, administrar bien el tiempo, tener una actitud optimista y ser sistemático en el estudio.

- Que los estudiantes de Ciencias Médicas aprovechen al máximo el tiempo de estudio desde el primer día de clases, de manera sistemática, así como también fomentar su participación en actividades con fines recreativos.

- Proponerse una meta desde el comienzo de la carrera, sin exigirse más de lo que se pueda alcanzar.

\section{Referencias bibliográficas.}

Barraza, A. (2014). El estrés académico en los alumnos de postgrado. PsicologíaCientífica.com. Disponible en: http://www.psicologiacientifica.com/estres-academico-postgrado/.

Barraza, A. (2015). Características del estrés académico de los alumnos de educación media superior. PsicologíaCientífica.com. Disponible en: 
http://www.psicologiacientifica.com/estres-academico-educacion-mediasuperior-2/.

Bedoya, S.A., Perea, M. \& Ormeño, R. (2016). Evaluación de niveles, situaciones generadoras y manifestaciones de estrés académico en alumnos de tercer y cuarto año de una Facultad de Estomatología [Versión electrónica]. Revista Estomatológica Herediana, 1 (16), 15 - 20.

Berrío, N. \& Mazo, R. (2011). Caracterización psicométrica del inventario de estrés académico en estudiantes de pregrado de la Universidad de Antioquia. Trabajo de grado para optar al título de psicóloga, Departamento de Psicología, Facultad de Ciencias Sociales, Universidad de Antioquia, Medellín, Colombia.

Caldera, J.F., Pulido, B.E. \& Martínez, M.G. (2017). Niveles de estrés y rendimiento académico en estudiantes de la carrera de Psicología del Centro Universitario de Los Altos [Versión electrónica]. Revista de Educación y Desarrollo, 7, 77-82.

Celis, J., Bustamante, M., Cabrera, D., Cabrera, M., Alarcón, W. \& Monge, E. (2001). Ansiedad y Estrés Académico en Estudiantes de Medicina Humana del Primer y Sexto Año [Versión electrónica]. Anales de la Facultad de Medicina, 1 (62), 25-30.

Feldman, L., Goncalves, L., Chacón-Puignau, G., Zaragoza, J., Bagés, N. \& De Pablo, J. (2008). Relaciones entre estrés académico, apoyo social, salud mental y rendimiento académico en estudiantes universitarios venezolanos [Versión electrónica]. Universitas Psychologica, 3 (7), 739-751.

González, J.L. (2001). Nosología psiquiátrica del estrés [Versión electrónica]. Psiquis, $22,1-7$.

Gutiérrez, Y.F., Londoño, K.F. \& Mazo, R. (2009). Diseño y validación del inventario de estrés académico en estudiantes de pregrado de la Universidad Pontificia Bolivariana de Medellín. Trabajo de grado para optar al título de psicólogo, Facultad de Psicología, Escuela de Ciencias Sociales, Universidad Pontificia Bolivariana, Medellín, Colombia.

Lazarus, R.S. \& Folkman, S. (1986). Estrés y procesos cognitivos. España: Ediciones Martínez Roca.

Martín, I. M. (2015). Estrés Académico en Estudiantes Universitarios [Versión electrónica]. Apuntes de Psicología, 1 (25), 87-99.

Martínez, E.S. \& Díaz, D.A. (2017). Una aproximación psicosocial al estrés escolar [Versión electrónica]. Educación y Educadores, 2 (10), 11-22. 
Misra, R. \& Castillo, L. (2004). Academic Stress Among College Students: Comparison of American and International Students [Versión electrónica]. International Journal of Stress Management, 2 (11), 132-148.

Pardo, J. (2017). Estrés en estudiantes de Educación Social [Versión electrónica]. Indivisa. Boletín de estudios e investigación, 9, 9-22.

Pellicer, O., Salvador, A. \& Benet, I. (2015). Efectos de un estresor académico sobre las respuestas psicológica e inmune en jóvenes [Versión electrónica]. Psicothema, 2 (14), 317-322.

Rodríguez, M. (s.f.). El estrés en el ámbito laboral. El estrés y sus causas. Disponible en: http://www.saudeetrabalho.com.br/download/estres-uso.pdf.

Román, C. A., Ortiz, F. \& Hernández, Y. (2015). El estrés académico en estudiantes latinoamericanos de la carrera de Medicina [Versión electrónica]. Revista Iberoamericana de Educación, 7 (46), 1-8.

Sandín, B. (1995). El estrés. En A. Bellock, B. Sandín y F. Ramos, Manual de Psicopatología (pp. 3-52). Madrid: McGraw-Hill.

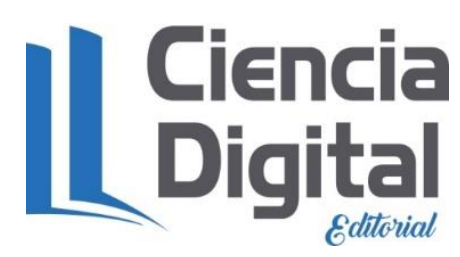


PARA CITAR EL ARTÍCULO INDEXADO.

Borges Sierra, L., Cairo Martínez, J. C., Pedroso González, M. M., \& Velastegui López, L. E. (2021). Afrontamiento al estrés académico en estudiantes de Ciencias Médicas . Anatomía Digital, 4(2), 170-180. https://doi.org/10.33262/anatomiadigital.v4i2.1718

\section{Ciencia}

El artículo que se publica es de exclusiva responsabilidad de los autores y no necesariamente reflejan el pensamiento de la Revista Anatomía Digital.

El artículo queda en propiedad de la revista y, por tanto, su publicación parcial y/o total en otro medio tiene que ser autorizado por el director de la Revista Anatomía Digital.
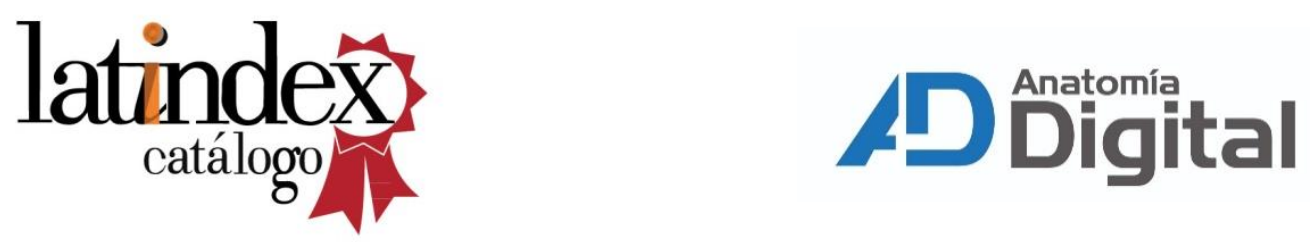